\title{
PANCREATITIS AGUDA: INDICE DE SEVERIDAD EN TC. EVALUACION DE COMPLICACIONES Y HOSPITALIZACION
}

Drs. Jaime Schwaner $C^{(1)}$, Francisco Rivas $B^{(2)}$, Adrián Cancino $N^{(3)}$, Osvaldo Torres $R^{(3)}$, Carlos Briceño $C^{(2)}$, Fernando Riquelme $P^{(2)}$.

1. Radiólogo Hospital Regional de Concepción.

2. Gastroenterólogo. Hospital Regional de Concepción.

3. Cirujano. Hospital Regional de Concepción.
Abstract: Objectives. We compare the classic tomographic criteria of Balthazar and the index of severity as a predictive factor for the development of complications and length of hospital stay. Material and method. Retrospective study of 61 patients with clinical diagnostic of acute pancreatitis to whom an abdominal CT was made, with an intravenous bolus injection of contrast material. These explorations were analyzed with the Balthazar criteria, pancreatic degree of necrosis and the index of severity of the CT. Later, we made a correlation with the appearance of complications and the hospital days. Result. The result was similar to other studies in the prediction of complications and length of hospital stay, but with less average days in our series and without mortality. Conclusión. We concluded that the pancreatic factor of necrosis and the index of severity in the tomographic study improve the valuation foretells of the acute pancreatitis.

Key words: Pancreatitis, CT.

Resumen: Objetivos. Analizar los criterios tomográficos clásicos de Balthazar y el índice de severidad (Balthazar clásico más necrosis), en pancreatitis aguda, como factores predictivos del desarrollo de complicaciones y tiempo de hospitalización en nuestra experiencia. Material y método. Estudio retrospectivo en 61 pacientes con diagnóstico clínico de Pancreatitis aguda a quienes se les realizó TC abdominal con contraste

Schwaner J. y cols. Pancreatitis aguda: Indice de severidad en TC. Evaluación de complicaciones y hospitalización. Rev Chil Radiol 2003; 9: 187-193.

Correspondencia: Dr. Jaime Schwaner $C$.

Servicio de Imagenología.

Hospital Guillermo Grant B. Concepción.

Jschwaner@entelchile.net endovenoso. Estas exploraciones se analizaron según los criterios de Balthazar, grado de necrosis pancreática e índice de severidad en TC. Posteriormente se realizó una correlación con la presencia de complicaciones y tiempo de hospitalización. Resultado. Los hallazgos fueron similares a otros estudios en la predicción de complicaciones y tiempo de estadía hospitalaria, pero en nuestra serie hubo menos días promedio de hospitalización y no tuvimos mortalidad. Conclusión. El factor de necrosis pancreática y el índice de severidad en el estudio tomográfico mejora la evaluación pronóstica de la pancreatitis aguda.

Palabras claves: Indice severidad, Pancreatitis aguda, TC.

\section{Introducción}

La pancreatitis aguda (PA) es una enfermedad inflamatoria que además del páncreas compromete tejidos vecinos y a distancia. Su presentación clínica es muy variable y en algunos casos tiene una morbimortalidad importante. Requiere de un diagnóstico precoz y un manejo oportuno para evitar complicaciones y muertes ${ }^{(1-3,7)}$.

La tomografía computada (TC) con contraste intravenoso ha demostrado ser de gran utilidad para el diagnóstico, reconocer complicaciones y evaluar el grado de inflamación y necrosis, estableciendo un pronóstico de severidad de la enfermedad. Tambiénse usa para guiar biopsias y drenajes percutáneos ${ }^{(4,5)}$.

En la evaluación pronóstica de la PA clásicamente se han utilizado los criterios clínicos de Ranson y los imagenológicos de Balthazar que toman en consideración los grados de inflamación. Actualmente se ha agregado el llamado índice de severidad descrito en 1990 por el mismo autor y que evalúa en conjunto el grado de inflamación pancreática y el porcentaje de necrosis glándular ${ }^{(6,8,9)}$. 
El objetivo de este trabajo es evaluar en nuestra experiencia los criterios clásicos de Balthazar y el índice de severidad en $\mathrm{TC}^{(6)}$ y su utilidad como factores predictivos para el desarrollo de complicaciones en PA y la prolongación de la hospitalización.

\section{Material y métodos}

Se realizó un análisis retrospectivo de 61 pacientes, 39 mujeres y 22 hombres, con PA, diagnosticada por clínica y exámenes de laboratorio, a quienes se les realizó TC abdominal. El periodo de estudio comprendió desde Septiembre del año 2000 a Agosto del año 2001. Las edades estaban comprendidas entre los 15 y 86 años, con un promedio de 51 años.

La TC se efectuó en un equipo CT MAX-640 de GE, se administró $1000 \mathrm{cc}$. de contraste oral y 100 cc. de contraste intravenoso en bolo e infusión. Se realizaron cortes de 5 y $10 \mathrm{~mm}$. antes y después de la inyección de contraste desde las bases pulmonares hasta la bifurcación aortica.

Las exploraciones fueron interpretadas y clasificadas según los criterios clásicos de Balthazar, se evaluó el porcentaje de necrosis y se asignó el índice de severidad.

Los criterios tomográficos clásicos descritos en 1985 por Balthazar(7) (Tabla I) clasifican las PA en cinco grados (A-B-C-D-E) en función al tamaño, contorno, densidad y existencia de enfermedad inflamatoria peripancreática con o sin la presencia de una o más colecciones líquidas (Figuras 1,2,3).

El índice de severidad de la $\mathrm{TC}^{(6)}$ combina los criterios de Balthazar con el porcentaje de necrosis de la glándula pancreática.

Se considera necrosis pancreática el área glándular que no realza después de la administración de contraste endovenoso ${ }^{(1-3,5,6)}$. Se clasificó según el porcentaje de la glándula afectada en tres grupos: 1) Menor al 30\%. 2) Menor al 50\%. 3) Mayor o igual al $50 \%$.

Se asignó un puntaje a cada grado de la clasificación clásica y a cada grupo de necrosis. Estos se suman obteniéndose el índice de severidad en TC que se clasifica en bajo (0-3 puntos); medio ( 4-6 puntos) y alto (7-10 puntos) (Tabla II) (Figuras 1,2,3,4).

\section{Resultados}

Las causas de PA encontradas en los pacientes estudiados fueron: Colélitiasis en 25 casos siendo la más frecuente; 3 casos de colelitiasis e ingesta de alcohol; 2 casos de colelitiasis más hiperlipidemia; 13 pacientes con diagnóstico desconocido o misceláneo; siendo esta la segunda causa en orden de frecuencia. En 10 casos la PA ocurrió en pacientes colecistectomizados, y en dos colecistectomizados más hiperlipidemia, en 3 casos había colédocolitiasis y se registro un caso de colangitis.

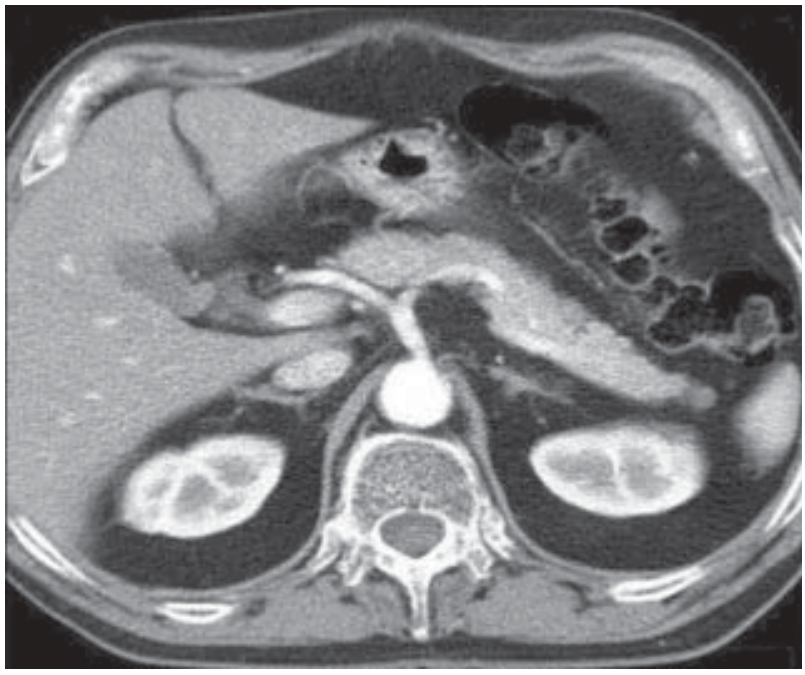

Figura 1. TC demostrando un páncreas de tamaño normal con impregnación homogénea en paciente con clínica de $P A$. Balthazar grado A. Indice de severidad bajo (0 punto)

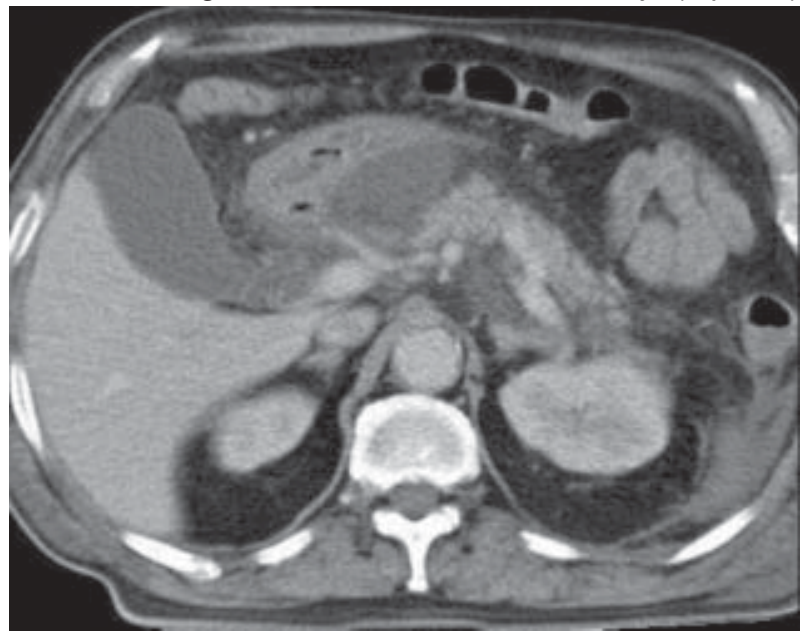

Figura 2. Páncreas aumentado de tamaño con necrosis de un $30 \%$ a nivel cefálico asociado a colección líquida en cabeza y en el espacio pararrenal anterior izquierdo. Grado D según Balthazar. Indice de severidad: medio (5 puntos).

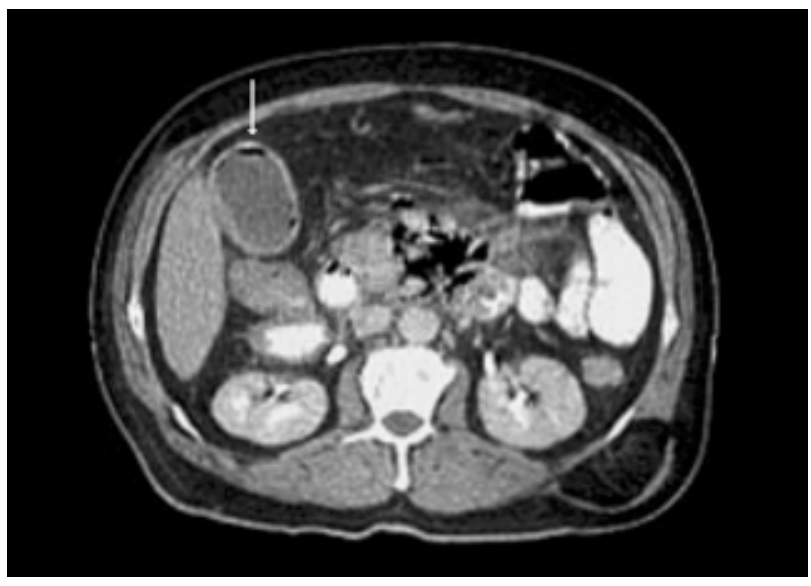

Figura 3. PA con importante presencia de gas en cuerpo del páncreas asociado a colección líquida mal definida. Grado $E$ de Balthazar. Nótese la presencia de gas intravesicular (flecha); este paciente requirió cirugía. 


\section{TABLA I. Criterios tomográficos clásicos de Balthazar}

GRADO A: Páncreas normal.

GRADO B: Aumento de tamaño focal o difuso del páncreas. Incluyendo: contornos irregulares, atenuación heterogénea del páncreas, dilatación del ducto pancreático, pequeñas colecciones líquidas dentro del páncreas, sin evidencia de enfermedad peri pancreática.

GRADO C: Alteraciones pancreáticas intrínsecas asociadas con: aumento de la densidad peripancreatica difusa y parcial, que representa cambios inflamatorios en la grasa.

GRADO D: Colección líquida única mal definida.

GRADO E: Dos o múltiples colecciones líquidas pobremente definidas o presencia de gas en o adyacente al páncreas.

TABLA II. Indice de severidad en TC

\begin{tabular}{lcrc} 
BALTHAZAR & Puntos & $\%$ NECROSIS & Puntos \\
\hline A & 0 & 0 & 0 \\
B & 1 & 30 & 2 \\
C & 2 & $30-50$ & 4 \\
D & 3 & $>50$ & 6 \\
E & 4 & &
\end{tabular}

\section{Suma de los puntos en TC= INDICE DE SEVERIDAD}
$0-3$
$4-6$
$7-10$
Bajo
Medio
Alto

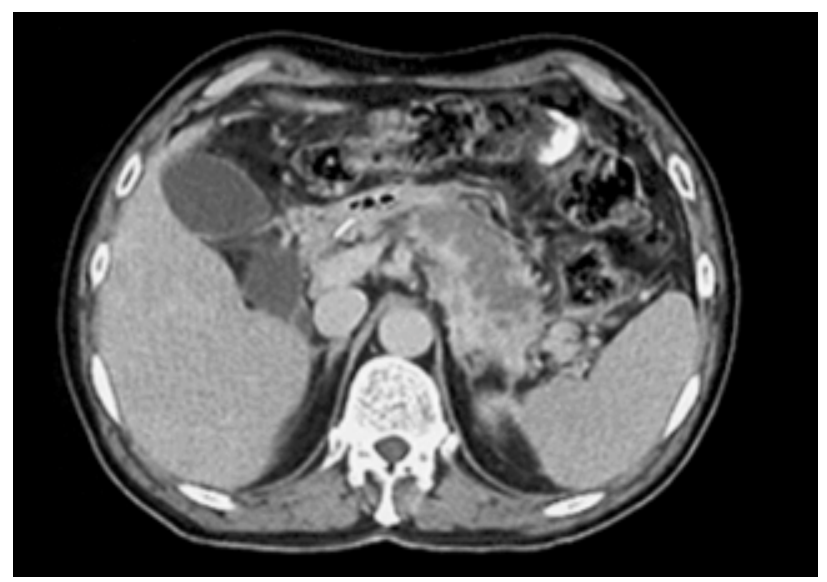

Figura 4. Páncreas aumentado de tamaño a nivel de cuerpo y cola con alteraciones intrínsecas y cambios inflamatorios de la grasa peripancreática. Necrosis mayor al $50 \%$ a nivel de cuerpo y cola. Balthazar grado C. Indice de severidad: alto (8 puntos).

En dos paciente solo se encontró el antecedente de ingesta alcohólica (Tabla III).

A todos los pacientes se le realiza TC abdominal en el lapso comprendido entre 1 a 10 días desde su ingreso. A 12 dentro los primeros 3 días y a 49 entre los 4 y 10 días. En pocos casos se efectuó 2 o 3 exámenes.
Del total de pacientes estudiados sólo 9 requirieron tratamiento quirúrgico (Figuras 3,6,7). La estadía media hospitalaria fue de 15 días con un rango de 1 a 138 días.

Se efectuó análisis de los días de hospitalización y complicaciones en relación a la clasificación clásica de Balthazar, grado de necrosis e índice de severidad.

Correlacionados los criterios tomográficos de Balthazar con los días de hospitalización observamos que, a mayor grado de compromiso inflamatorio de la glándula, aumentan en forma directa los días de hospitalización (Tabla IV).

Asociando la necrosis pancreática con los días de estadía hospitalaria vimos que a mayor necrosis de la glándula en general mayores son los días de hospitalización (Tabla V).

En cuanto al índice de severidad en TC, obtuvimos que la estadía hospitalaria más prolongada ocurre cuando mayor es el índice de severidad (Tabla VI).

De los 61 pacientes estudiados 10 (17\%) presentaron complicaciones, 7 mujeres y 3 hombres, con un rango de edad que oscilaba entre 22 y 69 años y un promedio de 45 años. Dentro de las complicaciones consignadas destacan 6 pacientes con seudoquiste infectado y operados; 1 paciente con seudoquiste (Figura 8 ) que requirió sólo control médico y 2 pacientes con necrosis pancreática infectada que requirieron cirugía. Sólo un paciente registró colangitis y fue operado.

Los días de estadía media en el hospital de estos pacientes fue de 66 días con una variación de 19 a 138 días, la mas prolongada correspondió a un paciente con seudoquiste infectado y operado con 97 días en UCl. No observamos complicaciones mortales en nuestro estudio.

Clasificando los pacientes complicados según Balthazar, necrosis pancreática, índice de severidad y días promedio de hospitalización obtuvimos el mayor número de pacientes en el grado $\mathrm{E}$ de Baltazhar, necrosis mayor de $50 \%$ e índice de severidad alto con 5 pacientes. Habían 4 pacientes en grado $D$, con necrosis menor del $50 \%$ e índice medio y sólo un paciente en grado $A$, sin necrosis e 


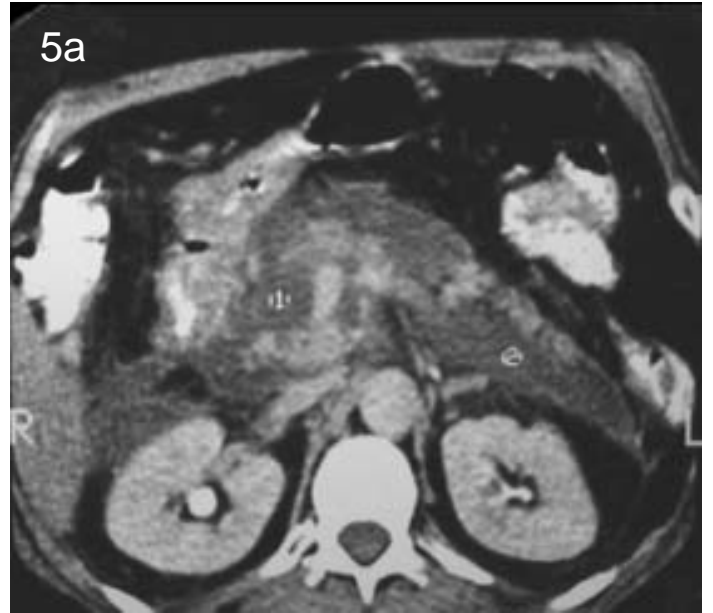

8
0
3
2
3
3
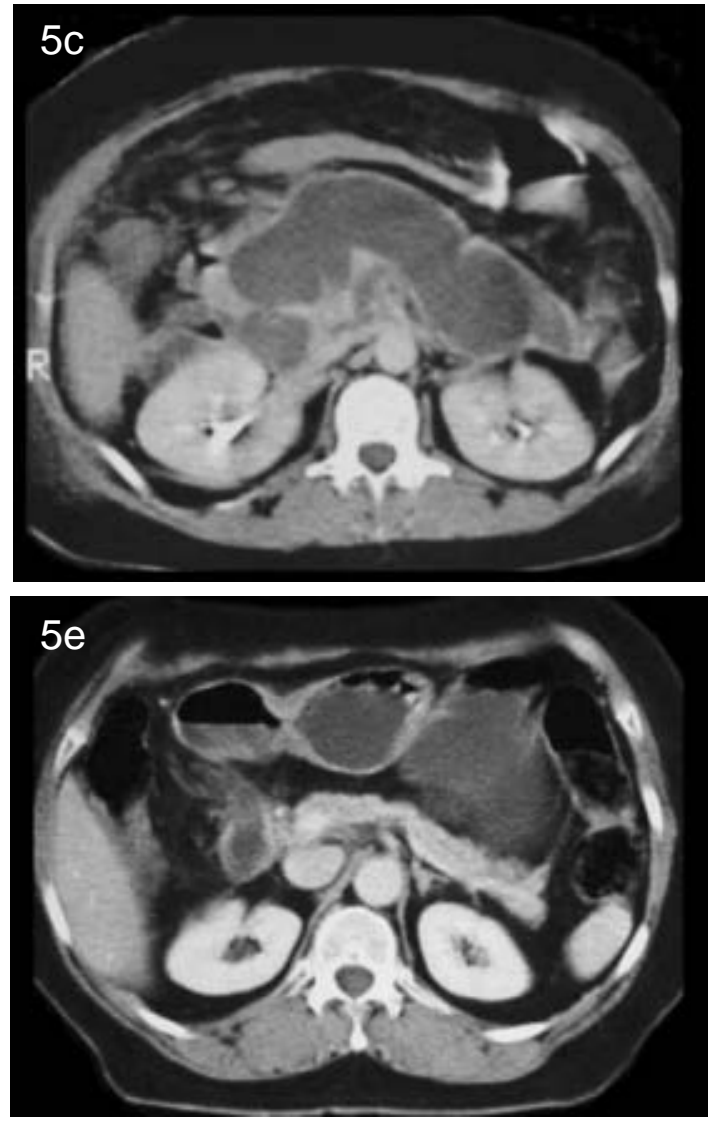

Figura 5 a-e. Mujer de 62 años con PA. a: Balthazar E al ingreso. b: Control a los 20 días de hospitalización. Se sospecha colección infectada y se realiza punción encontrándose Enterococo Aglomerans, se trata con antibióticos. c: Control a los 30 días, posterior al cual se opera, encontrándose biliperitoneo infectado con necrosis vesicular. Se realiza drenaje vesicular. d: Control a los 60 días de hospitalización. Por aumento de la infección peripancreática se reopera dejandose drenaje quirúrgico más antibióticos. Fístula pancreática y colección infectada. e: Control tomográfico a los 10 meses después de la PA. Páncreas de aspecto normal.

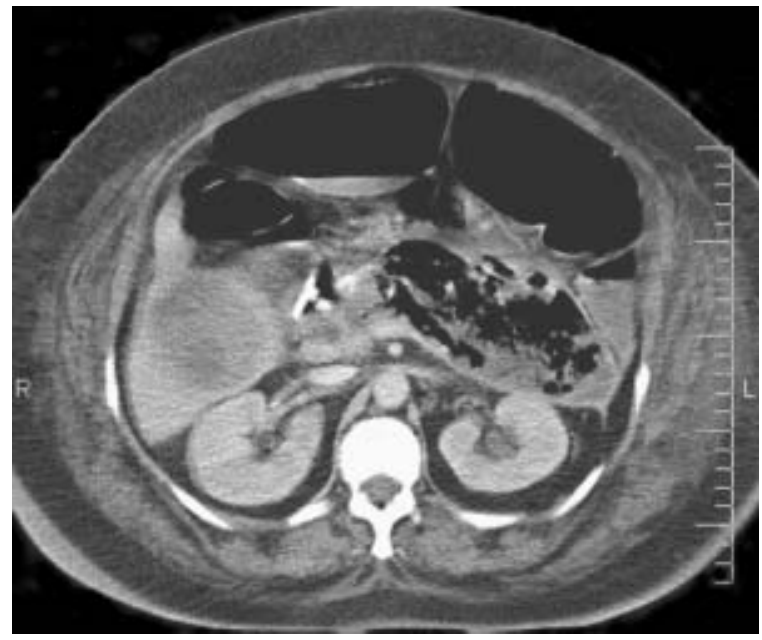

Figura 6. Mujer de 63 años con gran colección en cuerpo y cola del páncreas e importante presencia de gas en su interior. Balthazar grado E. Esta paciente requirió cirugía.

\begin{tabular}{|lr|}
\hline Tabla III. & \\
Causas & 25 \\
\hline Colélitiasis & 3 \\
Colélitiasis + alcohol & 2 \\
Colélitiasis + dislipidemia & 3 \\
Colédocolitiasis & 1 \\
Colangitis & 10 \\
Colecistectomizado & 2 \\
Colecistectomizado + dislipidemia & 2 \\
Alcohol & 13 \\
Desconocido o misceláneo & 61 \\
Total & \\
\hline
\end{tabular}




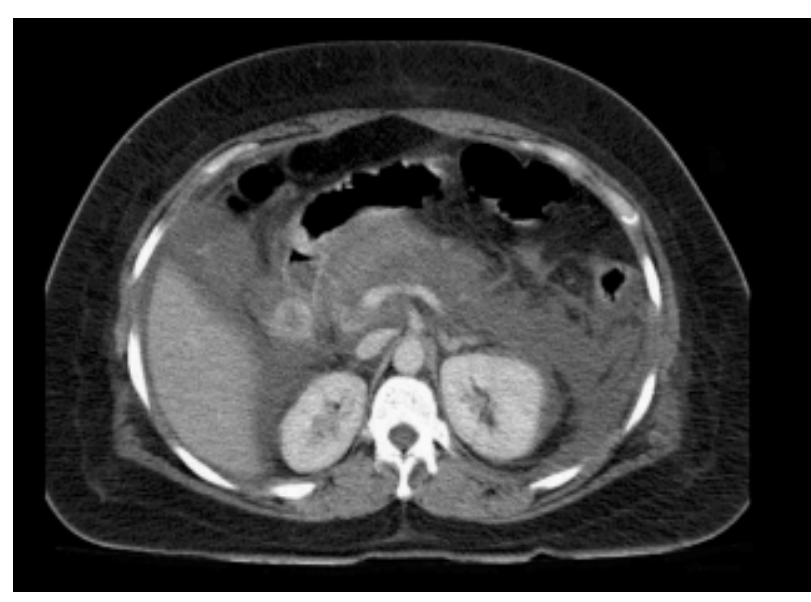

Figura 7. Mujer de 35 años con colección única mal definida Balthazar grado $D$. Necrosis pancreática mayor al 50\%. Indice de severidad alto (9 puntos).

Tabla IV. Grados de Baltazhar y hospitalización promedio

$\begin{array}{lcc}\begin{array}{l}\text { Baltazhar } \\ \text { (Grado) }\end{array} & \begin{array}{c}\text { Pacientes } \\ N^{\circ}(\%)\end{array} & \begin{array}{c}\text { Hospitalización } \\ \text { (días) }\end{array}\end{array}$

$\begin{array}{lrrr}\text { A } & 9 & (15 \%) & 12 \\ \text { B } & 15 & (24 \%) & 14 \\ \text { C } & 12 & (20 \%) & 22 \\ \text { D } & 16 & (26 \%) & 33 \\ \text { E } & 9 & (15 \%) & 56 \\ \text { TOTAL } & 61 & (100 \%) & -\end{array}$

Tabla VI. Indice de severidad y hospitalización promedio

\begin{tabular}{lccc}
$\begin{array}{l}\text { Indice de } \\
\text { severidad }\end{array}$ & $\begin{array}{c}\text { Pacientes } \\
\mathbf{N}^{\circ}(\%)\end{array}$ & $\begin{array}{c}\text { Hospitalización } \\
\text { (días) }\end{array}$ \\
\hline Bajo & 36 & $(59 \%)$ & 16 \\
Medio & 14 & $(23 \%)$ & 38 \\
Alto & 11 & $(18 \%)$ & 46 \\
TOTAL & 61 & $(100 \%)$ & \\
& & &
\end{tabular}

índice bajo en que la complicación resultó ser una colangitis (Tabla VII)

Dentro de estos cinco grupos la estadía más prolongada correspondió al grado D, ya que en este grupo se encontraba el paciente de más larga estadía (138 días) con más de 2 intervenciones quirúrgicas y un gran riesgo vital. Las complicaciones más graves, se observan en pacientes con mayor necrosis y mayor índice de severidad.

Clasificando los pacientes según morbilidad manifestada por seudoquistes y abscesos ${ }^{(6)}$ y relacionándola con el índice de severidad, obtenemos que con un índice de severidad de 0-3

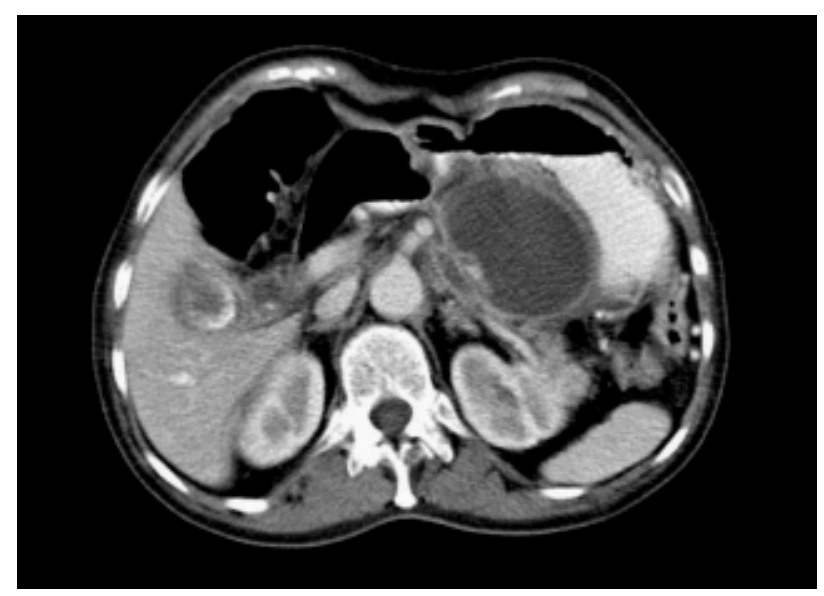

Figura 8. Seudoquiste pancreático a nivel del cuerpo que desplaza el estomago hacia anterior con una pared bien definida. Como hallazgo asociado se evidencia colélitiasis.

Tabla V. Porcentaje de necrosis y hospitalización promedio

$\begin{array}{ccc}\begin{array}{c}\text { Necrosis } \\ (\%)\end{array} & \begin{array}{c}\text { Pacientes } \mathrm{N}^{\circ} \\ (\%)\end{array} & \begin{array}{c}\text { Hospitalización } \\ \text { (días) }\end{array}\end{array}$

$\begin{array}{lrrr}\text { Sin necrosis } & 36 & (59 \%) & 16 \\ 30 \% & 12 & (20 \%) & 46 \\ >30-<50 \% & 8 & (13 \%) & 33 \\ >50 \% & 5 & (8 \%) & 52 \\ & & & \\ \text { TOTAL } & 61 & (100 \%) & -\end{array}$

puntos la morbilidad alcanza un $3 \%$, con un índice de severidad de 4-6 puntos alcanza un $29 \%$ de morbilidad y un $50 \%$ cuando el índice de severidad se encuentra en el rango de 7-10 puntos, debemos destacar que en nuestro estudio no tuvimos casos mortales.

De los pacientes con PA sin necrosis (36) sólo uno se complicó, lo que representa un índice de morbilidad de $3 \%$, en cambio aquellos con necrosis pancreática (25) se complicaron 9 lo que representa un $36 \%$ de morbilidad.

\section{Discusión}

La PA es una enfermedad de evolución impredecible que va desde estados asintomáticos hasta casos mortales. Evoluciona como pancreatitis intersticial edematosa y enfermedad autolimitada en aproximadamente un $70-80 \%$ de los casos y solo un $20-30 \%$ de los casos toma un curso severo ${ }^{(8,13)}$. En nuestro estudio los pacientes que se complicaron alcanzan un $16 \%$.

La frecuencia en USA de PA es de 270 casos por 100.000 habitantes en personas de 15-44 años y se eleva a 540 casos por 100.000 en personas mayores de 65 años.

Las causas más comunes, son los cálculos biliares y la ingestión de alcohol y ocurren en un 
TABLA VII. Complicaciones y hospitalización (promedio) según grado, necrosis y severidad (promedio)

\begin{tabular}{|c|c|c|c|c|c|}
\hline $\begin{array}{l}\text { Pac. complicados } \\
\text { (número) }\end{array}$ & $\begin{array}{l}\text { Balthazar } \\
\text { (Grado) }\end{array}$ & $\begin{array}{l}\text { Necrosis } \\
(\%)\end{array}$ & & $\begin{array}{l}\text { eridad } \\
\text { dice) }\end{array}$ & $\begin{array}{l}\text { Hospitalización } \\
\text { (días) }\end{array}$ \\
\hline 1 * & $\begin{array}{l}\text { A } \\
B \\
C\end{array}$ & 0 & 0 & (bajo) & 19 \\
\hline 4 & D & $30-50 \%$ & 5 & (medio) & 69 \\
\hline $\begin{array}{l}5 \\
* \text { Paciente con cola }\end{array}$ & $E$ & $>50 \%$ & 9 & (alto) & 63 \\
\hline
\end{tabular}

$60-80 \%$ de los casos. Otras causas incluyen trauma abdominal cerrado, trauma iatrogénico (trauma postoperatorio y colangiopancreatografia retrograda), hipertrigliceremia, hipercalcemia, drogas, etiología infecciosa, anomalías congénitas (páncreas divisum y quiste de colédoco), tumores pancreáticos o ampulares, anormalidades vasculares (embolía ateroesclerotica, hipoperfusión, vasculitis), fibrosis quistica y síndrome de Reye. Estas causa misceláneas ocurren en aproximadamente un 10\% de los casos. En un 10 a 25\% no se encuentra una causa $^{(5-10)}$.

En nuestra serie tuvimos como causa cálculos biliares (alcoholismo incluido), en 36 casos (59\%), 1 caso de colangitis y 3 casos de colédocolitiasis. En 13 pacientes no se consignó una causa lo que corresponde a un $21 \%$, un poco más alto que el porcentaje referido en la literatura ${ }^{(5-10)}$, lo que puede deberse a la dificultad de contar con exámenes de laboratorio completos, oportunos o muy específicos para algunos casos como la fibrosis quística y otros. Debemos mencionar que nuestros casos de PA ocurrieron en 12 pacientes colecistectomizados y que en algunos hubo más de un agente causal.

En la actualidad la TC con contraste nos permite demostrar compromisos que van desde un mínimo edema hasta grandes colecciones líquidas y necrosis pancreática que se desarrollan en los casos más graves $^{(1,2,6,8,9,14)}$.

El diagnóstico de PA leve se determina generalmente por hallazgos clínicos y de laboratorio.

El aporte de la TC es excluir otras patologías, confirmar el diagnóstico clínico de PA, evaluar la extensión del compromiso pancreático y estudiar las complicaciones ${ }^{(8)}$. Es también útil en el pronóstico de severidad, con una mayor efectividad que la clasificación de pancreatitis según Ranson ${ }^{(8,9,20)}$.

Para evaluar el pronóstico de las PA hemos utilizado los criterios clásicos de Balthazar (Tabla I) más el grado de necrosis pancreática definida como aquellas áreas del parénquima pancreático que se realzan entre 30-50 UH después de la inyección de contraste endovenoso (realce normal entre 50-150
UH) o realzan menos que el bazo, ya que normalmente ambos órganos sufren un realce similar $^{(1-3,8,11)}$. Este hallazgo es bien demostrado con técnica helicoidal.

Johnson(12) comunicó que la necrosis pancreática detectada en TC, con contraste endovenoso, se relaciona estrechamente con los hallazgos quirúrgicos con una sensibilidad y especificidad mayor al $90 \%$. Es necesario sin embargo hacer notar que la necrosis se puede manifestar algunos días después del episodio agudo y no ser detectada en una TC inicial.

En nuestra serie a medida que aumenta el grado de Balthazar, el grado de necrosis y el índice de severidad aumentaron las complicaciones y los días de hospitalización.

En el estudio original de Balthazar 1990(6), los pacientes sin necrosis pancreática tenían una hospitalización promedio de 25 días, lo que en nuestro estudio alcanzó a 16 días. Para los casos con un $30 \%$ de necrosis llega a 54 días en el estudio de Balthazar y 46 días en nuestros casos; para los casos de necrosis pancreática de $50 \%$ y mayor la estadía hospitalaria en el trabajo de Balthazar era 107 y 109 días respectivamente y en nuestra serie 33 y 52 días. Aun cuando observamos que a mayor grado de necrosis los días de hospitalización promedio aumentaron, fueron significativamente menor a los observados por Balthazar.

Posiblemente el desarrollo de nuevos antibióticos, más específicos y con mayor concentración en páncreas este acortando la hospitalización, unido al tratamiento en unidades de cuidado intensivo en forma precoz y al uso racional de la cirugía ${ }^{(18)}$.

En los casos que se demuestre necrosis infectada el tratamiento de elección será el drenaje percutáneo guiada por US, TC o quirúrgico(2-8-14-19).

En nuestro estudio el índice de morbilidad aumentó con relación al grado de severidad y especialmente en los casos de PA con necrosis, esto se relaciona con lo descrito por Balthazar en su trabajo original (Tabla VIII) aunque en menor grado y sin mortalidad en nuestro serie. 


\begin{tabular}{|c|c|c|c|}
\hline \multicolumn{2}{|c|}{$\begin{array}{l}\text { GRADO } \\
\text { (Puntos) }\end{array}$} & \multirow{2}{*}{$\begin{array}{c}\text { MORBILIDAD } \\
8 \%\end{array}$} & \multirow{2}{*}{$\begin{array}{c}\text { MORTALIDAD } \\
3 \%\end{array}$} \\
\hline Bajo & $0-3$ & & \\
\hline Medio & $4-6$ & $35 \%$ & $6 \%$ \\
\hline Alto & $7-10$ & $92 \%$ & $17 \%$ \\
\hline
\end{tabular}

Una limitación de nuestro estudio es que no todos los pacientes dignosticados clínicamente como PA, en nuestro hospital, fueron estudiados con TC de abdomen. Otra limitación fue el tiempo entre el diagnóstico y la realización del examen, debido a la dificultad del diagnóstico en algunos casos y a la disponibilidad de horas para TC de abdomen.

Creemos que los días de hospitalización en algunos casos no representa en forma segura el real índice de morbilidad por PA, desafortunadamente la información respecto a otras causas de morbilidad no fue registrada en las fichas que fueron revisadas en forma retrospectiva.

En conclusión en nuestra experiencia los resultados obtenidos validan el índice de severidad como predictor de complicaciones y hospitalizaciones más prolongadas.

\section{Agradecimientos}

A los Tecnólogos Médicos de la unidad de TC del HGGB: Ramón Lastra, Elizabeth Moya, María Muñoz e Iván Pizarro por su asistencia en la realización de los exámenes, como también en la obtención de las imágenes.

\section{Bibliografía}

1. Balthazar E. Acute pancreatitis: Assessment of severity with clinical and CT evaluation. Radiology 2002; 223:603-613.

2. Balthazar E, Freeny P, Van Sonnenberg E. Imaging and intervention in acute pancreatitis. Radiology 1994; 193:297-306.

3. Bradley E. A clinically based classification system for acute pancreatitis. Arch. Surg. 1993; 128:586-590.
4. Paulson E, Vitellas K, Keogan M. Acute pancreatitis complicated by gland necrosis: spectrum of finding on contrast-enhanced CT. AJR 1999; 172: 609-613.

5. Jacobs J., Birnbaum B. Computed tomography evaluation of acute pancreatitis. Seminars in Roentgenol 2001; 2: 92-98.

6. Balthazar E, Robinson D, Megibow A. Acute pancreatitis: value of $C T$ in establishing prognosis. Radiology 1990; 174: 331-336.

7. Balthazar E., Ranson J, Naidich D. Acute pancreatitis: prognostic value of CT. Radiology 1985; 156: 767-772.

8. Beltrán C, Berger Z, Gálvez S, Watkins G. Consenso Nacional sobre diagnóstico y tratamiento de la pancreatitis aguda. Rev. Chilena de medicina intensiva 2001; 16 (2): 100-113.

9. Echeverría F, Martínez B, López F. Criterios pronósticos de pancreatitis aguda. Importancia de la valoración de la necrosis pancreática mediante TC con contraste intravenoso. Radiología 1997; 39 (10): 685-691.

10. Díaz P, Sáenz R, Watkins G. Criterios pronósticos de Ranson y Balthazar y aplicabilidad de criterios de Atlanta 1992 en pancreatitis aguda grave. Gastr. Latinoamericana 1998; 9(2): 150-157.

11. White M, Wittenberg J, Mueller P. Pancreatic necrosis: CT manifestations. Radiology 1986; 158: 343-346.

12. Johnson C.D., Sthefens D, Saar M. CT of acute pancreatitis: correlation between lack of contrast enhancement and pancreatitis necrosis. AJR 1991; 156: 93-95.

13. Berger $\mathrm{H}$, and Isenmann R. Surgical management of necrotizing pancreatitis. Surgical clinics of North America. 1999; 79 (4): 783-800.

14. Sonnenberg E, Wittich G, Chon K et al. Percutaneous radiologic drainage of pancreatic abscesses. AJR 1997; 168: 979-984.

15. Lee, Sagel, Stanley, Heiken. Computed body with MRI correlation. Edición 1999; 873-953.

16. Vargas J, Díaz J, Csendes P. Clasificación actual de la pancreatitis aguda: Una aproximación clínico radiológica. Rev Chil Radiol. 2000; 6 (4): 137-139.

17. Lecesne R, Taourel P, Bret P. Acute pancreatitis: interobserver and agreement colangiopancreatography with outcome. Radiology 1999; 211: 727-735.

18. Cancino A, Torres O, Schwaner J. Tratamiento antibiótico de la pancreatitis aguda necrotizante infectada. Rev. Chile Cirugía. 2002; 54 (6): 600-604.

19. Niedmann J P. Tratamiento percutáneo de las complicaciones locales de la pancreatitis aguda. Gastr. Latinoamérica 2002; 13(3): 197-201.

20. Cruz F. Tomografía computada en Pancreatitis aguda. Gastr. Latinoamérica 2002; 13(3): 181-182. 